\title{
МАТЕМАТИЧЕСКОЕ ПРОГНОЗИРОВАНИЕ ПАРАМЕТРОВ ОПУХОЛЬ-СЕЛЕКТИВНОГО НАКОПЛЕНИЯ ПАРАМАГНИТНЫХ НАНОЧАСТИЦ КЛЕТКАМИ РЕТИНОБЛАСТОМЫ
}

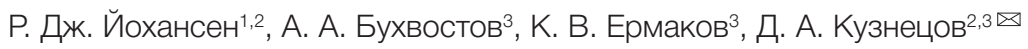 \\ Кафедра математики и компьютерных наук, Университет Южной Дании, Оденсе, Дания \\ Институт химической физики имени Н. Н. Семенова РАН, Москва \\ Кафедра медицинских нанобиотехнологий, Российский национальный исследовательский медицинский университет имени Н. И. Пирогова, Москва
}

\begin{abstract}
Ретинобластома - злокачественное новообразование, поражающее сетчатку глаза. Целью работы было разработать вычислительный подход к прогнозированию опухоль-специфического накопления наночастиц, высвобождающих катионы изотопов двухвалентных металлов $\left({ }^{25} \mathrm{Mg},{ }^{43} \mathrm{Ca},{ }^{60} \mathrm{Co},{ }^{67} \mathrm{Zn}\right.$, ...) в клетках ретинобластомы человека. Предложена математическая модель, основанная на применении уравнения Гомперца и оригинальной версии немарковской популяционной динамики. Она основана на факте ярко выраженного дискриминационного распределения препарата между злокачественными и «соседствующими» С ними нормальными клетками и различиях в параметрах их клеточных циклов. Учтены как фармакокинетические, так и фармакодинамические особенности наночастиц PMC16 - порфирин-производных фуллерена $\mathrm{C}_{60}$, известных благодаря их уникальным возможностям в отношении направленной доставки парамагнитных изотопов металлов в раковые клетки, сопровождающейся существенным химиотерапевтическим эффектом. Демонстрируя зависимость от скорости роста опухоли, но не от ее массы в стационарной фазе, рандомизированный уровень накопления препарата в клетках ретинобластомы формализован как ценный в прогностическом отношении расчетный метод, пригодный для оптимизации проводимых в настоящее время доклинических исследований катионообменных наночастиц РМС16.
\end{abstract}

Ключевые слова: ретинобластома, парамагнитные цитостатики, нанокатиониты, опухоль-селективное накопление наночастиц, математическая модель накопления препарата

Благодарности: эта работа была бы невозможна без помощи в технических вопросах, которую нам оказали сотрудники Университета Южной Дании в Оденсе в рамках программы Erasmus-Plus DK06811/2020. Авторы выражают особую благодарность Патриции Владычевской, главному руководителю SDS Erasmus.

$凶$ Для корреспонденции: Дмитрий Анатольевич Кузнецов

ул. Островитянова, д. 1, г. Москва, 117997; kuznano@mail.ru

Статья получена: 27.06.2018 Статья принята к печати: 18.08 .2018

DOI: $10.24075 /$ vrgmu.2018.078

\section{TOWARDS A COMPUTATIONAL PREDICTION FOR THE TUMOR SELECTIVE ACCUMULATION OF PARAMAGNETIC NANOPARTICLES IN RETINOBLASTOMA CELLS}

\author{
Johansen RJ1,2, Bukhvostov $\mathrm{AA}^{3}$, Ermakov KV³, *Kuznetsov DA ${ }^{2,3}$ 凶
}

Department of Mathematics and Computer Science, University of Southern Denmark, Odense, DK-5230, Denmark

${ }^{2}$ N. N. Semenov Institute of Chemical Physics, Russian Academy of Sciences, Moscow

${ }^{3}$ Department of Medical Nanobiotechnologies, N. I. Pirogov Russian National Research Medical University, Moscow

Retinoblastoma is a malignant growth affecting retina. An original combination of modified Non-Markov and Gompertzian computational approaches is proven of being a reliable tool for prediction of tumor selective accumulation of the bivalent metal isotopes $\left({ }^{25} \mathrm{Mg},{ }^{43} \mathrm{Ca},{ }^{60} \mathrm{Co},{ }^{67} \mathrm{Zn}, \ldots\right)$ - releasing nanoparticles in human retinoblastoma cells. This mathematical model operates with a starting point of the discriminative drug uptake caused by a gap-like distinction between the neighboring malignant and normal cell proliferation rates. This takes into account both pharmacokinetic and pharmacodynamic peculiarities of PMC16, fullerene- $\mathrm{C}_{60}$ based nanoparticles, known for their unique capabilities for a cancer-targeted delivery of paramagnetic metal isotopes followed by an essential chemotherapeutic effect. Being dependent on a tumor growth rate but not on the neoplasm steady state mass, a randomized level of drug accumulation in retinoblastoma cells has been formalized as a predictive paradigm suitable to optimize an ongoing PMC16 preclinical research.

Keywords: retinoblastoma, paramagnetic cytostatics, nanocationites, tumor selective nanoparticles uptake, drug accumulation mathematical model

Acknowledgments: this work was performed due to an exceptional technical assistance kindly provided by Erasmus-Plus DK06811/2020 Program associates affiliated with the Southern Denmark University at Odense, Denmark, and, most specifically, by Ms. Patricia Wladycziewski, SDU Erasmus chief supervising officer.

Correspondence should be addressed: Dmitry A. Kuznetsov

Ostrovityanova 1, Moscow, 117997; kuznano@mail.ru

Received: 27.06.2018 Accepted: 18.08 .2018

DOI: 10.24075/brsmu.2018.078 
Ретинобластома (РБ) человека очень чувствительна к некоторым парамагнитным изотопам металлов, индуцирующих магнитные изотопные эффекты, которые ингибируют репарацию ДНК в злокачественных клетках и способствуют образованию укороченных фрагментов ДНК, непригодных для репарации дефектов нуклеосомной ДНК [1-4]. Это свидетельствует в пользу перспективности нового подхода в химиотерапии РБ, основанного на применении наночастиц (НЧ), высвобождающих ${ }^{25} \mathrm{Mg}^{2+}$, ${ }^{43} \mathrm{Ca}^{2+},{ }^{60} \mathrm{Co}^{2+},{ }^{67} \mathrm{Zn}^{2+}, \quad$ на фоне гиперэкспрессии репарационных ДНК-полимераз $\beta[1,5,6]$.

Комплексы парамагнитных изотопов с РMC16 (рис. 1) специфический тип амфифильных низкотоксичных НЧ были разработаны в качестве транспортеров ионов, применяемых как в таргетной противоопухолевой терапии, так и для последующего контролируемого высвобождения препарата в клетках $[1,5]$. Влияние этих парамагнетиков проявляется в значительном снижении скорости пролиферации, что показано в экспериментах на клеточных линиях РБ Y79 и WERI-RB-1 [2-4].

Согласно статистике PubMed, количество публикаций, посвященных использованию НЧ для пассивной адресной доставки лекарств, увеличилось за последние 15 лет с 40 (2000 г.) до почти 1800 (2015 г.), причем НЧ рассматриваются только в контексте терапии солидных опухолей [7]. Предполагается, что в ходе доклинических испытаний будет показана возможность использования РМС16 в отношении РБ. Однако ряд эпигенетических факторов, характерных для данного типа опухоли, не позволяет a priori судить о селективном поглощении таких H4 [8-10].

Таким образом, надежный метод прогнозирования объемов селективного накопления НЧ (PMC16) в РБ может быть полезен для исследований, в которых изучается возможность применения данного препарата в борьбе с этим видом онкологических заболеваний.

Целью данной работы было предпринять попытку разработать такой метод с использованием инструментов математического моделирования.

Следует отметить, что математический подход к разработке сценария доклинических и клинических испытаний новых лекарств уже практиковали в экспериментальной онкологии и близких к ней областях [11-17].

\section{МАТЕРИАЛЫ И МЕТОДЫ}

Для моделирования использовали конвенциональные значения объемов распределения НЧ в клетках и параметры их митотических циклов для нормальной и РБизмененной тканей сетчатки глаза (таблица) $[8,9,15,18]$ Эти данные обрабатывали с помощью алгоритма Sigma QXL600 в анализаторах HP9107 (Hewlett-Packard, Inc; США) и Olivetti Riccetta SL110 (Ing. C. Olivetti \& Со.; Италия). Использовали модифицированный метод вероятностной аппроксимации Пенмана-Дальбрё [14, 16], позволяющий синхронно процессировать решения составленных на основе данных эксперимента и литературных данных модифицированных систем уравнений немарковской популяционной динамики $[12,14]$ и рутинной системы уравнений Гомперца [11].

\section{РЕЗУЛЬТАТЬ}

Цитотоксичное действие препаратов на здоровые ткани, резистентность к ним опухолевых клеток и опухоль- селективное накопление лекарств - критически важные проблемы, возникающие при химиотерапии [5, 6, 8]. Методы математического моделирования могут помочь в выборе и анализе возможных путей, применимых для решения этих проблем [7, 14, 17, 18]. В некоторых случаях такой подход помогает прогнозировать уровень чувствительности к фазоспецифичным средствам (НЧ) с учетом их невекторального, «пассивного», распределения в клеточном пуле, состоящем из клеток, делящихся с разными скоростями и характеризующихся разными величинами продолжительности интерфаз их митозов.

\section{Немарковская популяционная динамика}

Динамику популяций с разными параметрами клеточных циклов, которые подвергаются воздействию лекарственных средств, можно моделировать следующим наиболее простым способом:

$$
x(t)=\lambda x(t-\tau)[1-\mathrm{D}(t)],
$$

где $x(t)$ - плотность популяции в заданный момент времени $t, \lambda-$ скорость обновления клеток, $\tau-$ время генерации, $D(t)-$ фактор влияния среды, т. е. $D(t)>0-$ достижение эффективной концентрации препарата в системе. Согласно этой модели, время элиминации популяции злокачественных клеток $\left(T_{m}\right)$ и сопредельной поддерживающей популяции (незлокачественных) клеток $\left(T_{\mathrm{h}}\right)$, при коэффрициенте элиминации $Z$, отражающем эфффективность лечения:

$$
Z=1-T_{m} / T_{h} .
$$

Эфффективность лечения является немонотонной функцией отношения между временем генерации клеток и длительностью курса лекарственной терапии при достижении максимума в момент, когда продолжительность генеративного цикла ограничивающих клеток кратна продолжительности химиотерапии. Дальнейший анализ показывает, что в полностью периодических системах при условии, что $\tau>\delta>\tau / 2$, время элиминации $T$ должно быть:

$$
T=\tau \omega /|\tau-(\delta+\omega)| .
$$

Здесь $\delta$ - продолжительность периода, в течение которого лекарство производит ожидаемый эффект, а $\omega$ - период, в рамках которого дозировка препарата не позволяет

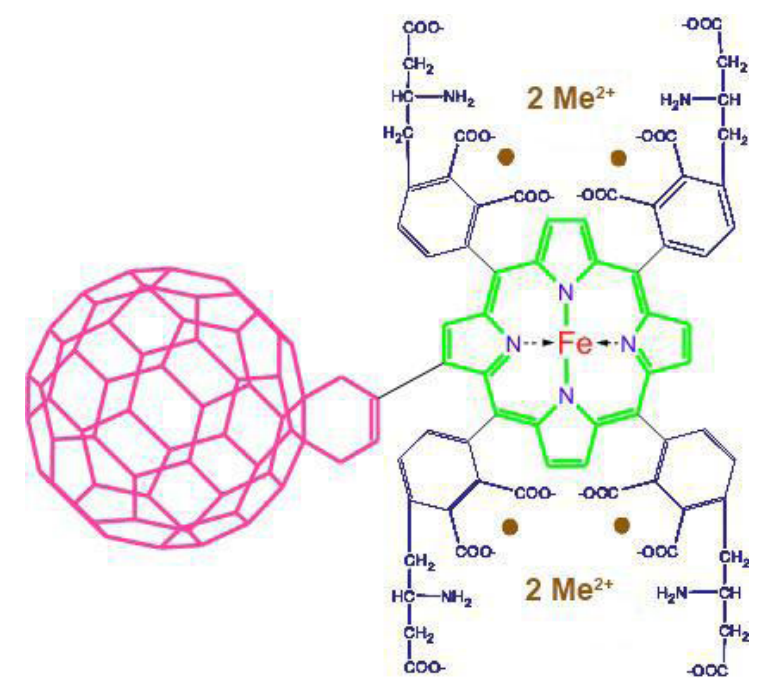

Рис. 1. Структура наночастиц РМС16 (циклогексил(Со $)_{60}$ порфирин), переносящих $\mathrm{Me}^{2+}$ и обладающих выраженными мембранотропными/ амфиифильными свойствами [1] 
Таблица. Параметры обновления клеточных популяций в культурах клеток РБ человека Y79 и WERI-RB-1

\begin{tabular}{|c|l|c|c|}
\hline Параметр & \multicolumn{1}{|c|}{ Значение } & Т, 4 & Источник \\
\hline$T G_{1}$ & Продолжительность фазы $G_{1}$ & 8,0 & {$[9,15]$} \\
\hline$T_{S}$ & Продолжительность фазы $S$ & 7,5 & {$[8,15]$} \\
\hline$T_{G_{2} M}$ & Продолжительность фазы $G_{2} M$ & 2,0 & {$[8,18]$} \\
\hline$T_{G_{0}}$ & Продолжительность фазы $G_{0}$ & 3,0 & {$[9,15]$} \\
\hline$T_{\text {Aрорtosis }}$ & Продолжительность фазы апоптоза & 4,0 & {$[8,9]$} \\
\hline
\end{tabular}

ему оказывать воздействие. $\tau=\delta+\omega-$ точка, в которой значение $T$ бесконечно.

Исходя из этого можно предположить, что классическая немарковская модель популяционной динамики $[12,14]$ позволяет моделировать распределение Н4 (РМС16) между злокачественной (быстрорастущей) и здоровой (медленнорастущей) частями клеточного пула. Упоминавшийся ранее амфифильный фармакофор (РМС16, транспортер изотопов бивалентных металлов на основе циклогексил-порфина- $\mathrm{C}_{60}$; рис. 1) является подходящим объектом для немарковской модели: ранее была показана способность НЧ этого типа индуцировать цитостатический эффекк в клетках острого миелобластного лейкоза и РБ $[1,5,6]$.

Предложенная нами двухрезервуарная (бикомпартментная) модель вполне соответствует представленным ниже фармакокинетическим параметрам (применимы в немарковской модели); межвидовые и рандомизированные влияния на $\mathrm{CL}, \mathrm{V}, \mathrm{Q}$ и V2 скорректированы с учетом остаточной ошибки $[12,13,16]$. Эта модель позволяет прогнозировать как селективное поглощение РМС16 клетками опухоли, обусловленное их быстрой пролиферацией, так и обычные фармакокинетические параметры.

\section{Межвидовые ковариантные модели}

\section{А. Фармакокинетическая модель}

$$
C=D / V \cdot\left[\frac{\left(\alpha-k_{21}\right)}{(\alpha-\beta)} \exp -(\alpha \cdot t)+\frac{\left(k_{21}-\beta\right)}{(\alpha-\beta)} \cdot \exp -(\beta \cdot t)\right]
$$

Б. Невекторная ковариантная модель

$$
\begin{gathered}
C L_{j}=\left[\theta_{3} \cdot O C C 1+\theta_{4}(W T-75)\right] \cdot \exp \left(\eta_{C L_{j}}\right) \\
V_{c j}=\left[\theta_{1}-(G F R-80) \cdot \theta_{2}\right] \cdot \exp \left(\eta_{V_{j}}\right) \\
k_{12_{j}}=\left(\theta_{5}\right) \cdot \exp \left(\eta_{k 12_{j}}\right)
\end{gathered}
$$

В. Модель популяционной динамики

$$
C_{i j}=\frac{D_{i v}}{\theta_{1} \cdot O C C_{1}+\theta_{2} \cdot(W T-75) \cdot \exp \left(\eta_{V_{j}}\right)}\left\{\begin{array}{l}
\frac{k_{21}-\frac{C L_{j}}{V_{v j}} \cdot \exp -\left(\frac{C L_{j}}{V_{c j}} t\right)}{\beta-\frac{C L_{j}}{V_{c j}}} \\
+\frac{k_{21}-k_{12}}{C L_{j}} \cdot \exp -\left(k_{12} f\right) \\
\frac{V_{c j}}{V_{j}} \beta
\end{array}\right.
$$

Г. Популяционные параметры

\section{Гомперцианская модель}

$$
\begin{gathered}
\theta_{1}=19,5 \\
\theta_{2}=0,198
\end{gathered}
$$

Модели, основанные на функции Гомперца, использовали для описания динамики роста раковых опухолей [7, 11, 14], а также в целях оптимизации стратегий лечения, включающих в себя антиангиогенную $[11,12]$ и лучевую терапию [11, 13].

Модель является детерминистической. Длительности фаз клеточного цикла $\tau_{\varphi}$ дискретизированы в нескольких элементарных возрастных интервалах $\alpha \in\left\{1, \ldots, N_{\phi}\right\}$, где $N_{\phi}$ - целое число, такое как $\tau_{\phi}=d t \cdot N_{\phi}$. Здесь $d t-$ временной шаг модели клеточного цикла. Плотность клеток $n_{\alpha, \phi}$ возраста $\alpha$ в фазе ф определяется по формуле:

$$
\frac{\partial n_{\alpha, \varphi}}{\partial t}+\nabla \cdot\left(v n_{\alpha, \varphi}\right)=P_{\alpha, \varphi} .
$$

В этом уравнении $\phi \in\left\{G_{1}, S, G_{2} M, G_{0}\right.$, апоптоз $\}$, а $\alpha \in$ $\left\{1, \ldots, N_{\phi}\right\} . P_{\alpha, \phi}$ - время роста плотности клеток в фазе $\phi$ возраста $\alpha$, следуют из общей модели клеточного цикла. Здесь внутриклеточные и внеклеточные условия были определены для клеток в конце фазы $G_{1}$.

Далее, принимая во внимания, что $\sum_{\alpha, \phi} n_{\alpha, \phi}$ постоянны, мы получаем описание поля давления:

$$
-\nabla \cdot(k \nabla p)=\sum_{\alpha, \varphi} P_{\alpha, \varphi} .
$$

Компьютерная обработка начинается с учета изначального распределения соседствующих клеток РБ и TP (ткань ретины/нормальной ткани) в каждом состоянии $\{\alpha, \phi\}$. Расчеты выполнены с применением метода разделения функций. Модель клеточного цикла для одного временного шага $d t$, дает новые значения $n_{\alpha, \phi}$. Уровень $P_{\alpha, \phi}$ определяется в результирующей системе:

$$
\left\{\begin{array}{c}
\frac{\partial n_{\alpha, \varphi}}{\partial t}+\nabla \cdot\left(v n_{\alpha, \varphi}\right)=0 \\
\frac{\partial n_{\alpha, \varphi}}{\partial t}+v \cdot \nabla n_{\alpha, \varphi}=\left(\sum_{\alpha^{\prime}, \varphi^{\prime}} P_{\alpha^{\prime}, \varphi^{\prime}}\right) n_{\alpha, \varphi}
\end{array}\right.
$$

Показателен вариант ее преобразования после применения к ключевым параметрам митотических циклов (см. таблицу), представленных в рамках немарковской модели популяционной динамики, отражающей череду возобновляемых состояний, биологически определенных как фазы $\left(G_{1}, S, G_{2}\right.$ и $\left.M\right)$ :

$$
\left\{\begin{aligned}
\frac{\partial n_{i}(t, x)}{\partial t}+\frac{\partial n_{i}(t, x)}{\partial t} & +d_{\tau}(t, x) n_{\tau}(t, x)+K_{i \rightarrow i+1}(t, x) n_{i}(t, x)=0 \\
n_{\tau+1}(t, 0) & =\int_{0}^{\infty} K_{i \rightarrow i+1}(t, x) n_{i}(t, x) d x \\
n_{1}(t, 0)= & 2 \int_{0}^{\infty} K_{I \rightarrow 1}(t, x) n_{I}(t, x) d x .
\end{aligned}\right.
$$

Здесь начальные условия - $\left(n_{i}(t=0)\right)_{1 \leq i \leq l}$.

Скорости гибели клеток в фазах обозначены как $d_{\phi}$; скорость же перехода между фазами принимается как периодически воспроизводимая. $K_{i \rightarrow i+1}$. Фаза $i(1 \leq i \leq l)$ может быть одной из классических четырех $\left(G_{1}, S, G_{2}\right.$ или M); также она может представлять собой аггрегированную фазу, например $S-G_{2}$, или фазу пролиферации $G_{1}-S$ $G_{2}-M$. C другой стороны, $n_{i}(t, x)$ может быть «субфразой», например до или после ограничения в фазе $G_{1}$. Уравнение описывает «эволюцию» значений плотности клеток $n_{\phi}(t, x)$, имеющих возраст $x$ во время $t$ в фразе $i$.

Системы, представленные выше, описывают две соседние клеточные популяции - быстрорастущую и медленнорастущую; физически они изолированы друг от друга, но являются «соседствующими». Поэтому в данной системе уравнений предполагается введение показателя 
эффективности противоопухолевого препарата (g) и функции цитотоксичности $(f)$ :

$$
g(D, t)=l l\left(1+\cos \left(2 \pi \frac{\left(t-\varphi_{B}\right)}{24}\right)\right) \cdot \frac{\left(D^{\Upsilon, B}\right)}{D_{50}^{\Upsilon, B}+D^{\Upsilon, B}},
$$

где $\lambda, v, \varepsilon_{D}, \alpha, B_{\max }, H, \varphi_{B}, Y_{B}, D_{50}-$ положительные константы, определенные на кривых динамики увеличения массы опухоли, взятые из литературных данных $[8,15,18]$ или вычисленные иными способами.

Разница в реакциях этих двух популяций клеток (пара РБ-ТР) на препарат выражается как $\varphi_{A}-\varphi_{B}=134$.

Возвращаясь к исходным положениям Гомперца, видим, что затухающая гармоническая аппроксимация отражает динамику популяции здоровых клеток (ТР):

$$
\left\{\begin{array}{c}
\frac{d P}{d t}=-\lambda P+\frac{i(t)}{V_{d i s t}} \phi(t) \\
\frac{d C}{d t}=-\mu C+\varepsilon_{C} P \\
\frac{d P}{d t}=\{-\alpha-f(C, t)\} Z-\beta A+Y \\
\frac{d A}{d t}=Z-Z_{e q}
\end{array}\right.
$$

где

$$
f(C, t)=F\left(1+\cos \left(2 \pi \frac{t-\varphi_{A}}{24}\right)\right) \frac{C^{y, A}}{C_{50}^{Y, A}+C^{Y, A}},
$$

а $\lambda, \mu, \varepsilon_{C}, \alpha, \beta, Y, Z_{e q}, F, \varphi_{A}, Y_{A}, C_{50}-$ положительные константы, определенные из кривых динамики увеличения массы опухоли [7, 10, 15].

Эти уравнения отражают диффузию и выведение лекарства согласно кинетике первого порядка (Р и С) для однократного внутривенного введения препарата. Здесь мгновенная доставка лекарства $i(t)$ и Ф (функция «открыто/ закрыто») соответствуют условиям гомеостаза здоровых тканей в линейной системе, устойчиво ссокусированной на $Z_{e q}, A_{e q}=\beta^{-1}\left(Y-\alpha Z_{e q}\right)$. Вмешательство цитотоксичного препарата увеличивает значение коэффициента саморегуляции $\alpha$.

Таким образом, наша модель, выведенная из ряда уравнений Гомперца, вполне подходит для прогнозирования динамики популяций опухолевых клеток:

$$
\left\{\begin{array}{c}
\frac{d P}{d t}=-\lambda P+\frac{i(t)}{V_{d i s t}} \phi(t) \\
\frac{d D}{d t}=-v D+\varepsilon_{D} P \\
\frac{d B}{d t}=-\alpha B \ln \left(\frac{B}{B_{\max }}-g(D, t) B\right) .
\end{array}\right.
$$

Этот подход - не что иное как способ представить изменения в составе клеточной популяции, прибегая К простому линейному аппроксимированию, обычно исключающему ответные реакции на введение эффектора (лекарства), если оно не приводит к увеличению объема «резервуара» клеток, т. е. массы опухоли:

$$
\left\{\begin{array}{c}
\frac{\partial}{\partial t} \mathrm{p}(t, x)+\frac{\partial}{\partial x} \mathrm{p}(t, x)+\{\mu+K(x)\} \mathrm{p}(t, x)=0 \\
\mathrm{p}(t, x=0)=2(1-f) \int_{\varepsilon \geq 0} K(\varepsilon) \mathrm{p}(t, \varepsilon) d \varepsilon \\
\mathrm{p}(t, x=0)=\mathrm{p}_{0}(x) \\
\frac{d}{d t} Q(t)=2 f \int_{\varepsilon \geq 0} K(\varepsilon) \mathrm{p}(t, \varepsilon) d \varepsilon-v Q(t) \\
Q(0)=Q_{0}
\end{array}\right.
$$

Акцентируя внимание на перспективах практического применения этой модели, следует указать на связь между цитостатическим эффектом и удельным объемом накопления препарата, выражающуюся в прерывании процесса пролиферации (плотность $p(t, x)$ ) в момент, соответствующий уровню клеточной популяции $Q(t)$ при наличии мишени для действия препарата $f$; для скорости прекращения митоза $Q$, где $f$ обладает высокой аффинностью к цитостатику.

\section{ОБСУЖДЕНИЕ}

\section{Селективное поглощение НЧ клетками опухоли. Вероятность и прогноз}

Итак, вероятностный прогноз объемов селективного поглощения НЧ клетками РБ преимущественно зависит от соотношения скоростей пролиферации злокачественных и здоровых клеток, тогда как величиной массы злокачественной ткани per se (количеством клеток РБ) можно практически пренебречь (рис. 2). Это следует из предсказуемого ответа клеток на быструю диффузию PMC16 in situ в момент появления нанопереносчиков $\mathrm{Me}^{2+}$ на границе раздела РБ/ТР. Однако в этом стохастическом сценарии «содружество» клеток лишь частично отражает энергетический ланшафт клеток-мишеней [16, 19], что делает вероятность захвата клетками наночастиц зависимой от подвижности энергетического ландшафта и, следовательно, от скорости увеличения объема наиболее быстрорастущей компоненты в паре РБ/ТР (рис. 3).

Изменение цветности от синего к красному на рис. 2 и 3 отражает тенденцию к преимущественному накоплению НЧ в наиболее быстрорастущем «резервуаре» в составе пары РБ/ТР.

Возвращаясь к вероятностному подходу [12, 14], можно сказать, что селективное накопление препарата с РМС16 в опухоли становится предсказуемым ввиду огромной разницы в скоростях увеличения тканевой массы РБ и ТР [8-10, 12]. Таким образом, прогнозируемое в нашей модели (рис. 2) поглощение РМС16, зависящее от скорости пролиферации, описывается уравнением:

$$
A_{C}=K d\left[\operatorname{tg} \alpha_{R B} /\left(\operatorname{tg} \alpha_{R B}-\operatorname{tg} \alpha_{R T}\right)\right],
$$

где $K_{d}$ - постоянная аппроксимации Арно-Пито [16, 19].

\section{«Волшебная пуля» Пауля Эрлиха: мечта или кошмар?}

В 1908-1913 гг. Пауль Эрлих предложил концепцию «волшебной пули» $[8,17]$, которая ознаменовала закат Вирховианской теории общей патологии. Сегодня эта концепция принята в контексте разработки средств адресной доставки лекарственных препаратов [5,

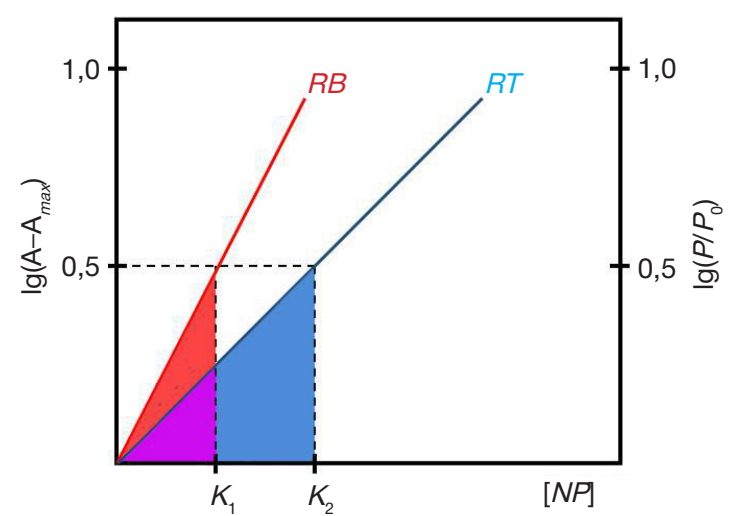

Рис. 2. Прогнозирование селективного поглощения НЧ при полной доступности внутриклеточных лигандов. $P-[N P]$ внутриклеточная концентрация поглощенных НЧ, количество массовых единиц на клетку;

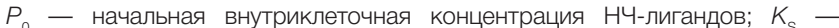
вектор $K$ уравнения Гомперца; $K_{1}-$ константа поглощения НЧ клеткой при достижении равновесного состояния; $K_{2}-$ эффективная константа насыщения пула клеточных лигандов при НЧ $\rightarrow 0,5 \mathrm{P}_{\max }$ 


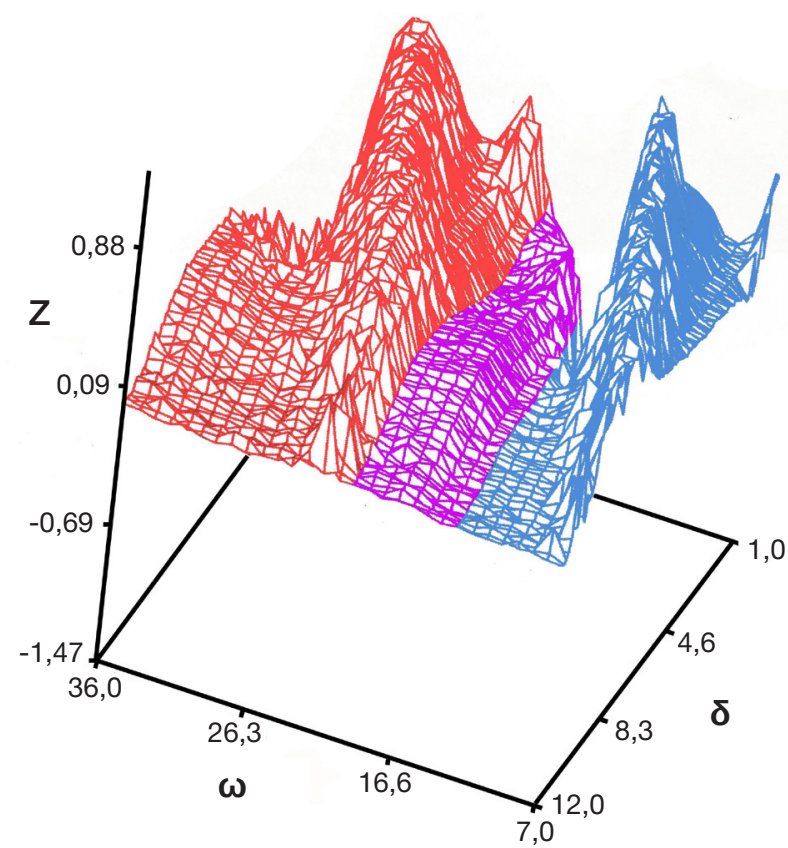

Рис. 3. Вероятностная модель распределения НЧ между соседствующими клетками РБ и ТР как функция дискриминационного отбора на основе различий между параметрами клеточных циклов. Z - коэффициент элиминации для злокачественных и окружающих здоровых клеток, определенный для времени

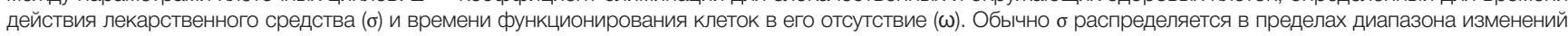
$\sigma=\sigma / 10$, где $\omega$ остается постоянным, в то время как внутренняя скорость появления «новорожденных» клеток составляет $\lambda=2$ при начальном объеме популяции $\times(0)=5$

7, 19]. Такие средства подразумевают использование наноструктур (частиц, контейнеров), т. е. разнообразных «волшебных пуль», которые не только доставляют препараты в клетки-мишени, но и обеспечивают затем их программируемое высвобождение в них [1, 5, 17].

Однако «стрелок», вооруженный «волшебными пулями", не видит полной картины. Так, к границе опухоли может быть доставлено достаточное количество молекул или ионов лекарства, что означает хороший выстрел, но теперь его результат во многом зависит от селективности поглощения этих молекул/ионов опухолевыми клетками. Таким образом, решающее значение имеет не столько точность траектории «полета» пули, сколько последующее распределение НЧ между соседствующими «резервуарами» злокачественных и здоровых клеток. Поэтому предложенная нами прогностическая модель может стать полезным инструментом для оптимизации алгоритмов доклинических и клинических исследований НЧ типа РМС16.

При введении препарата через шлеммов канал и/или интраокулярно (пути, часто используемые в терапии РБ [8]) такой подход не только возможен, но и предпочтителен. Побочные эфффекты, которые обычно возникают при парентеральном введении лекарств, можно не учитывать, когда приоритетом является химиотерапия РБ.

\section{ВЫВОДЫ}

Предложенная математическая модель позволяет прогнозировать объем селективного накопления лекарственных катионообменных НЧ в клетках ретинобластомы человека, если эти НЧ являются амфифильными и мембранотропными и обладают способностью проникать в клетку-мишень. Этим критериям полностью соответствуют представители семейства РМС16, созданные на основе порфириновых производных фуллерена $\mathrm{C}_{60}$, способные переносить и высвобождать катионы двухвалентных металлов. Модель, учитывающая различия в скоростях пролиферации здоровых и злокачественных клеток, может эффективно применяться для разработки и оптимизации программ доклинических исследований РМС16 и подобных им фармакофоров.

\section{Литература}

1. Buchachenko AL. Magneto-Biology and Medicine. New York: Nova Biomedical Publ., 2015.

2. Bukhvostov AA, Dvornikov AS, Ermakov KV, Kurapov PB, Kuznetsov DA. Retinoblastoma: magnetic isotope effects might make a differece in the current anti-cancer research strategy. Acta Medica (Prague). 2017; 60 (2): 93-6.

3. Bukhvostov AA, Dvornikov AS, Ermakov KV, Kuznetsov DA Retinoblastoma case: shall we get a paramagnetic trend in chemotherapy? Arch Cancer Res. 2017; 5 (4): 158-62.

4. Bukhvostov AA, Pavlov KA, Ermakov KV, Sidoruk KN, Rybakova IV, Kuznetsov DA, Roumiantsev SA. An atypical $\beta$-like DNA Polymerase of retinoblastoma cells as a target for spin-selective inhibitory cytostatics. J Fund Med Biol (Russian). 2018; 7 (2): 50-3.

5. Orlova MA, Osipova EY, Roumiantsev SA. Effect of ${ }^{67} \mathrm{Zn}$ -

nanoparticles on leukemic cells and normal lymphocytes. Brit $J$ Med Med Res. 2012; 2 (1): 21-30.

6. Orlov AP, Orlova MA, Trofimova TP, Kalmykov SN, Kuznetsov DA The role of zinc and its compounds in leukemia. J Biol Inorg Chem. 2018; 23 (3): 347-62.

7. Siccardi M, Owen A. Towards a computational prediction of nanoparticle pharmakokinetics and distribution. J In Silico \& In Vitro Pharmacol. 2016; 2 (1): 8-11.

8. Augsburger JJ, Chow CML, Dyer V, Roussel MF. Translating science into survival. In: Cajjar A, Pappo A, editors. St. Jude's Children's Research Hospital Report. St. Judes CRH Publ.: Memphis, TN - Cincinnati, OH. 2016; 6-55.

9. Zhang J, Benavente CA, McEvoy J, Flores-Otero J, Ding L, Chen X, Ulyanov A. A novel retinoblastoma therapy from genomic and 
epigenetic analyses. Nature. 2012; 481 (7381): 329-34.

10. Bozic I, Nowak MA. Resisting resistance. Ann Rev Cancer Biol. 2017; 1 (1): 203-21.

11. Bassukas ID. Comparative Gompertzian analysis of alterations of tumor growth patterns. Cancer Res. 1994; 54 (16): 4385-92.

12. Byrne $\mathrm{H}$, Prezidosi $\mathrm{L}$. Modelling solid tumor growth using the theory of mixtures. Math Med Biol. 2003; 20 (4): 341-66.

13. Komarova NL. Mathematical modelling of tumorigenesis: mission possible. Curr Opinion Oncol. 2005; 17 (1): 39-43.

14. Trapp S, Horobin RW. A predictive model for the selective accumulation of chemicals in tumor cells. Eur Biophys J. 2005; 34 (7): 959-66.

15. Udvardi L, Lakatos J, Loewenhaupt RK. Dividing Cell. In Vitro-In Silico Models. Szeged, Budapest: Alba Regia, 2017.

16. Lehman RJ, Waugh TS, Rattenau KR, Bielka H. An expanding compartment mode to implement a guest probe diffusion input adopted by the comprehensive Gunault muxtures theory. In: Sieliwanowicz B, Martell SJ, edsitors.Combinational Dynamics in Systems Theory. Sydney-Melbourne-Perth: Adler \& Adler Publ., 2018; 116-37.

17. Lamprecht A, Pellecker J. Anti-cancer nano-size agents: targeting paths and pharmacokinetics. In: Lamprecht A, editor. Nanotherapeutics. Drug Delivery Concepts in Nanoscience. NY-London-Singapore: Pan Stanford Publ., 2018; 92-101.

18. Altinok A, Gonze D, Levi F, Goldbeter A. An Automaton model for the cell cycle. Interface Focus. 2011; 1 (1): 36-47.

19. Delbreaux J, Pitot CA. Mathematical model in a new drug preclinical trial. Predictive power and limitations. LeuvenGhent-Antwerp: Leuven University Press, 2018.

\section{References}

1. Buchachenko AL. Magneto-Biology and Medicine. New York: Nova Biomedical Publ., 2015.

2. Bukhvostov AA, Dvornikov AS, Ermakov KV, Kurapov PB Kuznetsov DA. Retinoblastoma: magnetic isotope effects might make a differece in the current anti-cancer research strategy. Acta Medica (Prague). 2017; 60 (2): 93-6.

3. Bukhvostov AA, Dvornikov AS, Ermakov KV, Kuznetsov DA. Retinoblastoma case: shall we get a paramagnetic trend in chemotherapy? Arch Cancer Res. 2017; 5 (4): 158-62.

4. Bukhvostov AA, Pavlov KA, Ermakov KV, Sidoruk KN, Rybakova IV, Kuznetsov DA, Roumiantsev SA. An atypical $\beta$-like DNA Polymerase of retinoblastoma cells as a target for spin-selective inhibitory cytostatics. J Fund Med Biol (Russian). 2018; 7 (2): 50-3.

5. Orlova MA, Osipova EY, Roumiantsev SA. Effect of ${ }^{67} \mathrm{Zn}$ nanoparticles on leukemic cells and normal lymphocytes. Brit $J$ Med Med Res. 2012; 2 (1): 21-30.

6. Orlov AP, Orlova MA, Trofimova TP, Kalmykov SN, Kuznetsov DA. The role of zinc and its compounds in leukemia. J Biol Inorg Chem. 2018; 23 (3): 347-62.

7. Siccardi M, Owen A. Towards a computational prediction of nanoparticle pharmakokinetics and distribution. J In Silico \& In Vitro Pharmacol. 2016; 2 (1): 8-11.

8. Augsburger JJ, Chow CML, Dyer V, Roussel MF. Translating science into survival. In: Cajjar A, Pappo A, editors. St. Jude's Children's Research Hospital Report. St. Judes CRH Publ.: Memphis, TN - Cincinnati, OH. 2016; 6-55.

9. Zhang J, Benavente CA, McEvoy J, Flores-Otero J, Ding L, Chen X, Ulyanov A. A novel retinoblastoma therapy from genomic and epigenetic analyses. Nature. 2012; 481 (7381): 329-34.

10. Bozic I, Nowak MA. Resisting resistance. Ann Rev Cancer Biol. 2017; 1 (1): 203-21.

11. Bassukas ID. Comparative Gompertzian analysis of alterations of tumor growth patterns. Cancer Res. 1994; 54 (16): 4385-92.

12. Byrne $H$, Prezidosi L. Modelling solid tumor growth using the theory of mixtures. Math Med Biol. 2003; 20 (4): 341-66.

13. Komarova NL. Mathematical modelling of tumorigenesis: mission possible. Curr Opinion Oncol. 2005; 17 (1): 39-43.

14. Trapp S, Horobin RW. A predictive model for the selective accumulation of chemicals in tumor cells. Eur Biophys J. 2005; 34 (7): 959-66.

15. Udvardi L, Lakatos J, Loewenhaupt RK. Dividing Cell. In Vitro-In Silico Models. Szeged, Budapest: Alba Regia, 2017.

16. Lehman RJ, Waugh TS, Rattenau KR, Bielka H. An expanding compartment mode to implement a guest probe diffusion input adopted by the comprehensive Gunault muxtures theory. In: Sieliwanowicz B, Martell SJ, edsitors.Combinational Dynamics in Systems Theory. Sydney-Melbourne-Perth: Adler \& Adler Publ., 2018; 116-37.

17. Lamprecht A, Pellecker J. Anti-cancer nano-size agents: targeting paths and pharmacokinetics. In: Lamprecht A, editor. Nanotherapeutics. Drug Delivery Concepts in Nanoscience. NY_London-Singapore: Pan Stanford Publ., 2018; 92-101.

18. Altinok A, Gonze D, Levi F, Goldbeter A. An Automaton model for the cell cycle. Interface Focus. 2011; 1 (1): 36-47.

19. Delbreaux J, Pitot CA. Mathematical model in a new drug preclinical trial. Predictive power and limitations. LeuvenGhent—Antwerp: Leuven University Press, 2018. 